\title{
Self-consistent gyrokinetic determination of the electrostatic potential of rotating magnetic islands in tokamaks
}

\author{
M. Siccinio*, E. Poli*, F. J. Casson*, W. A. Hornsby**, \\ A. G. Peeters** \\ *Max-Planck-Institut für Plasmaphysik, Boltzmannstrasse 2, \\ D-85748 Garching bei München, Germany \\ **University of Bayreuth, 95440 Bayreuth, Germany
}

July 28,2011

\begin{abstract}
The electrostatic potential related to a magnetic island structure with imposed width and rotation frequency is studied by means of gyrokinetic simulations, which allow its self-consistent determination via the Poisson equation. An adiabatic response of the trapped ions at the island separatrix leads to a significant smoothing of the potential with respect to analytic calculations based on a complete flattening of the pressure profile inside the island. As a consequence, the magnitude of the polarization current is drastically reduced. When the island size is comparable to the ion banana width, the adiabatic response covers the whole island region, leading to no density flattening for islands rotating in the electron diamagnetic direction. This confirms previous results based on drift-kinetic simulations.
\end{abstract}

\section{Introduction}

Tearing modes are mainly radial magnetic perturbations which through magnetic reconnection lead to the appearance of magnetic islands, thus destroying the nested magnetic surfaces typical of tokamak equilibria at particular radial locations. Within magnetic islands, the plasma confinement is strongly reduced by virtue of the fast parallel particle streaming along the island radial magnetic field (equilibrium density and temperature profiles are internally flattened to a large degree). For this reason, and for their role as precursors 
in disruption events, tearing modes have been studied since the very beginning of the nuclear fusion research. The theory of the stability of the tearing mode was originally developed $[1,2]$ in a slab configuration, i.e. neglecting the effects of the curved magnetic geometry (this description is often referred to as "classical"). However, subsequent investigations showed both theoretically $[3,4]$ and experimentally $[5,6]$ that, in a typical tokamak configuration, tearing modes can be driven unstable even when the current profile is classically stable. For sufficiently large islands the dominant drive is represented by the suppression of the neoclassical bootstrap current in the island region caused by the aforementioned flattening of the pressure profile. This mechanism is often indicated as "neoclassical drive", and the corresponding modes are named "neoclassical tearing modes" (NTMs). Later, a number of other phenomena have been discovered to affect the island dynamics, especially for "small" islands. It has been shown, in fact, that there exists a threshold for the "seed" island size, below which the tearing mode is strongly damped and therefore it quickly disappears. Many different mechanisms have been suggested to explain this nonlinear threshold. In particular, a reduced degree of the flattening, and subsequently a lower neoclassical drive, has been pointed out $[7,8,9]$. In addition, the electrostatic potential associated with the rotation of the island with respect to the surrounding plasma has been shown to generate a polarization current $[10,11,12,13]$. Although other effects connected to the electrostatic potential have also been considered [14, 15, 16, 17], it is a common assumption to consider the stability of small magnetic islands to be dominated by polarization current and neoclassical drive [18].

In the cited papers, the potential was derived in the limit of large magnetic islands (compared to the particle orbits), assuming complete density flattening inside the island itself. Other works $[19,20,21]$ contain a self-consistent determination of the electrostatic potential (mainly in order to relax the condition $E_{\|}=0$ around the separatrix), neglecting however the effects connected to the toroidicity, in particular the contribution of trapped particles. Moreover, the assumption of complete pressure flattening was mantained. In this paper, we calculate for the first time the electrostatic potential related to a rotating magnetic island in toroidal geometry from a numerical solution of the relevant gyrokinetic equations and of the Poisson equation. The differences we find between our self-consistent simulations and the simplified models cited above are shown to strongly affect both the polarization current and the neoclassical drive, and by consequence the whole problem of the small island stability. The paper is structured as follows: in section 2, the model employed is described. In section 3 , the electrostatic potential associated with the presence of a rotating magnetic island in absence of equilibrium gradients is studied, evidencing an adiabatic response of trapped ions which in turn entails a deviation from the condition $E_{\|}=0$. In section 4, the repercussions of the phenomena discussed in section 3 on the polarization current are presented. In section 5, equilibrium gradients are included, and a relation between the degree of flattening of 
the pressure gradient (connected in turn with the neoclassical drive) and the electrostatic potential is estabilished. Conclusions are drawn in section 6 .

\section{Model}

In this paper, the physics of magnetic islands is investigated by means of gyrokinetic simulations. We employ a gyrokinetic Vlasov code, GKW [22], which solves the gyrokinetic equations in the so called flux-tube approximation $[23,24]$. The computational domain is defined as a "tube" built along a magnetic field line. The gradients of equilibrium quantities are supposed to be constant in the domain (linear equilibrium profiles). The gyrokinetic equations for both ions and electrons are solved. The perturbed fields (expressed through the electrostatic potential $\phi$ and the perturbed vector potential $A_{\|}$) are calculated self-consistently by means of the gyrokinetic Poisson and Ampère equations $[25,26]$, respectively. In this paper we do not address the self-consistent island evolution, thus Ampère equation is not solved, and a perturbed vector potential corresponding to a rotating magnetic island is imposed at every timestep. The code employs Hamada coordinates [27], namely a flux-surface label $\psi$ (playing the role of a radial coordinate, with $\psi=0$ at the centre of the computational box), a poloidal angle $\theta$ and a toroidal angle $\varphi$, defined in such a way that the corresponding contravariant components of the equilibrium magnetic field are functions of $\psi$ only. It is convenient to introduce the binormal coordinate

$$
\zeta=q(\psi) s-\gamma,
$$

where $s=\theta / 2 \pi$ (having fixed $s=0$ on the outer midplane of the tokamak), $\gamma=\varphi / 2 \pi$ and $q$ is the safety factor. The computational box perpendicular to the chosen field line is identified by the directions $\nabla \psi$ and $\nabla \zeta$ (which are linearly independent but not necessarily orthogonal) for every given point along $s$. Perturbations are assumed to be periodic on the $\psi-\zeta$-plane in both directions, and the equations are written in the Fourier space for these coordinates. A rotating magnetic island is conveniently described by a perturbation on the parallel vector potential in the form

$$
A_{\|}=\hat{A}_{\|} \cos (\xi)
$$

where $\hat{A}_{\|}$is a constant, according to the well-known constant- $\psi$ approximation [1]. The helical angle $\xi$ is defined as

$$
\xi=2 \pi(m s-n \gamma)-\omega t,
$$

where $m$ and $n$ are the poloidal and toroidal mode number, respectively, and $\omega$ is the island rotation frequency. NTMs can be shown to develop on magnetic surfaces, called rational (or resonant) surfaces, where the helicity of the magnetic field lines matches the one of the mode, i.e. $q=m / n$. A convenient choice for the representation of a magnetic island in the flux-tube limit is therefore to 
set $q(\psi=0)=m / n$, thus constructing the flux-tube around a field line lying on the rational surface. One can check by expressing $\xi$ in terms of $\psi$ and $\zeta$ and Taylor-expanding $q$ around $\psi=0$ that the mode in Eq.(2) is in general not periodic on the computational box in both $\psi$ and $\zeta$ directions. In order to treat $A_{\|}$as a perturbation (from the numerical point of view), a convenient approximation of Eq.(2) that fulfils the periodicity constraints has therefore been derived and implemented. The interested reader is referred to $[28,29]$ for details.

In this paper, we study the response of the plasma in the presence of a imposed magnetic island structure (i.e. both the island width $w$ and the island rotation frequency $\omega$ are treated as input parameters, and kept constant during each simulation), retaining FLR and finite banana width effects. The electrostatic potential (connected to the different ion and electron response to the rotation of the island with respect to the surrounding plasma) is calculated self-consistently by means of the gyrokinetic Poisson equation. The results of the simulation are presented in a $x, y$ plane, where $x$ and $y$ are lengths (normalized to the thermal ion Larmor radius $\rho_{L}$ ) measured along the $\nabla \psi$ and $\nabla \zeta$ directions, respectively. The point $x=0, y=0$ identifies the centre of the domain, and the line $x=0$ identifies the rational surface. The magnetic island considered is the $m=3, n=2$ mode, which fixes $q(x=0)=1.5$. The width of the box in the $x$ direction must be sufficiently larger than the island width, in order to avoid unphysical effects due to the periodicity constraints (e.g. a too fast decay of the island electrostatic potential, which would lead to unphysical electric fields).

In previous studies $[10,11]$, the analytical expression of the electrostatic potential was derived from the assumption of electrons fast enough to short out any parallel electric field, namely

$$
E_{\|}=-\nabla_{\|} \phi_{0}-\frac{1}{c} \frac{\partial A_{\|}}{\partial t}=0 .
$$

Writing the parallel gradient as

$$
\nabla_{\|}=\left.k_{\|} \frac{\partial}{\partial \xi}\right|_{\Omega},
$$

where $k_{\|}=-k_{\theta} x / L_{s}$, with $k_{\theta}$ the poloidal wavevector of the mode and $L_{s}$ the shear length, while

$$
\Omega=\frac{2 x^{2}}{w^{2}}-\cos \xi
$$

is a flux-surface label in presence of a magnetic island, and recalling that

$$
w=\sqrt{\frac{4\left|\hat{A}_{\|}\right| L_{s}}{B_{0}}}
$$


( $B_{0}$ being the total magnetic field strength), one obtains for the potential the well-known expression [10]

$$
\phi_{0}=\frac{\omega B_{0}}{k_{\theta} c}[x-h(\Omega)] .
$$

We employ the subscript 0 to indicate this particular expression. In Eq.(8), the function $h(\Omega)$ is an integration constant, whose form is determined from boundary conditions. It is important to stress the fact that fixing the form of $h(\Omega)$ implies the imposition of some conditions on the electron transport in the island region $[11,21]$. This point will be addressed in detail later on. A common choice for the function $h(\Omega)$ corresponding to complete flattening inside the island is [10]

$$
h(\Omega)=\frac{w}{\sqrt{2}}[\sqrt{\Omega}-1] \Theta(\Omega-1) .
$$

Such expression is characterised by a Heaviside step function $\Theta(\Omega-1)$ (i.e. $\Theta(x)=1$ if $x>0$ and 0 otherwise, while $\Omega=1$ identifies the island separatrix). Consequently, $\phi_{0}$ leads to a radial electric field which is constant inside the island (corresponding in particular to an $\mathbf{E} \times \mathbf{B}$ rotation with frequency $\omega$, as the plasma within the island rotates with it), and vanishing outside, exhibiting a singular derivative at the island separatrix (introduced by the derivatives of the Heaviside function) [12]. This discontinuity is strictly connected with the polarization current contribution, as addressed in detail in section 4 .

In our numerical model, the electrostatic potential and the particle distribution are calculated self-consistently, including the correct response to the perturbed magnetic geometry, in particular around the island separatrix. Collisions have been neglected.

\section{Electrostatic Potential - Neglecting Equilib- rium Pressure Gradients}

In this section, we first present the results of GKW simulations performed taking flat density and temperature profiles. In this case, the electrostatic potential, at least at a first glance, does not appear different from the analytical $\phi_{0}$ (Eq.(8)). However, a careful analysis shows that there are indeed some deviations. This allows us to point out some physical effects, whose importance will be discussed in the following.

As mentioned before, most of the existing analytical models of the tearing mode assume a complete flattening of the pressure profile inside the island region $[10,11,19]$. This would imply that in the simplified limit of flat equilibrium density and temperature profiles, no perturbation to those quantities should be observable. Actually, in our simulations the density profile in the presence 

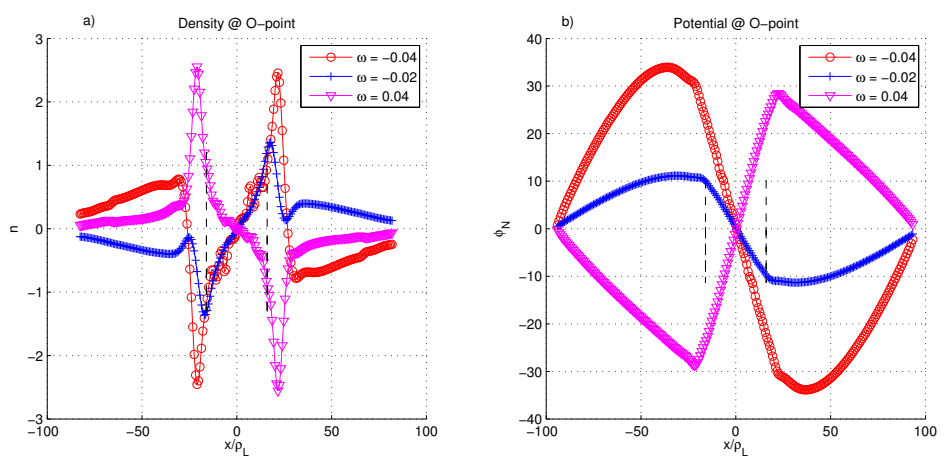

Figure 1: (a) Ion perturbed density profiles across the island O-point for $\hat{w}=16$, where $\hat{w}=w / \rho_{L}$ ( $\rho_{L}$ being the thermal ion Larmor radius) at different island rotation frequencies (b) Potential profile across the island O-point for $\hat{w}=16$ at different island rotation frequencies. Perturbed densities $\tilde{n}$ are normalized as $\tilde{n} /\left(n_{0} \rho_{*}\right)$, where $n_{0}$ is the equilibrium reference value, $R$ the tokamak major radius and $\rho_{*}=\rho_{L} / R$. Similarly, the potential is normalized to $e \phi / \rho_{*} T_{R}$, where $e$ is the fundamental charge and $T_{R}$ is the reference temperature for the normalization. Frequencies are normalized to the ion transit frequency, namely $v_{\mathrm{th}, i} / R, v_{\mathrm{th}, i}$ being the thermal ion velocity. With the parameters employed for the simulation presented, $\rho_{*}=2.7 * 10^{-3}$.

of the island has the shape shown in Fig.1. Such behaviour with peaks at the island separatrix is found in GKW simulations for a wide survey of frequencies and island widths. It was shown by a recent paper of Bergmann et al.[17] that where the scale of variation of the electrostatic potential is comparable to or smaller than the thermal ion banana width, trapped particles tend to respond adiabatically to the potential itself (an effect already discussed in relation to fast particle orbits in Ref.[14]). It is noted here that an ion adiabatic response in the limit of $w \ll \rho_{L}$ was already known from slab geometry calculations $[21,30]$.

The fact that the perturbed density $\delta n$ we observe in our simulations is indeed due to the aforementioned ion adiabatic response is supported by its linear scaling with $\omega$, (analogously to the potential, see Fig.1), and by the GKW velocity space diagnostic (Fig.2), which shows that the perturbed distribution $\delta f$ for the ions is entirely localized in the trapped region of the phase space.

This adiabatic response of the trapped ions has a feedback effect on the electrostatic potential. Around the island separatrix, the ion density perturbation can be approximated in the form

$$
\delta n_{i} \approx-\epsilon^{3 / 2} n_{0} \frac{e \phi}{T_{i}} .
$$

In contrary to the ions, we can suppose the dynamics of electrons to be dom- 

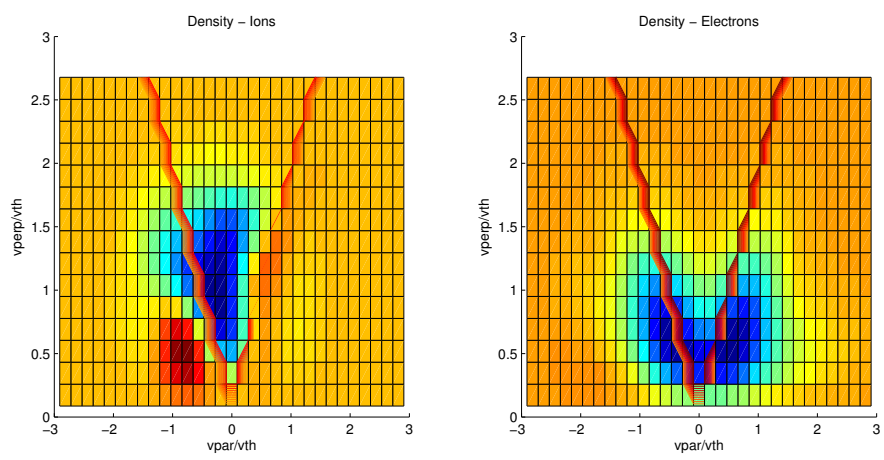

Figure 2: Perturbed distribution (averaged on $s$, i.e. along a magnetic field line) as a function of the velocity space coordinates at the island separatrix in correspondence with the O-point (i.e. $x=-\hat{w}, \xi=0$ ) for ions and electrons. Here, $\omega=-0.02$ and $\hat{w}=8$. The triangle separates the trapped domain (inside) from the passing one (outside). It is apparent that the ion response is well inside the trapped region of the phase space, while the electrons are mainly outside it.

inated by the parallel streaming along the field lines, as depicted in Fig.2 (the dominant balance, from a fluid point of view, being the one between the parallel gradient of the electron pressure and the parallel electric field, i.e. $\left.\nabla_{\|} n_{e}=-e E_{\|} / T_{e}\right)$. This means

$$
\delta n_{e} \approx n_{0} \frac{e \hat{\phi}}{T_{e}}
$$

(having supposed $\phi=\phi_{0}+\hat{\phi}$, which recalling Eq.(8) leads to $E_{\|}=-\nabla \hat{\phi}$ ). Neglecting for the sake of simplicity the FLR effects in the quasineutrality condition, we impose $\delta n_{i} \approx \delta n_{e}$, obtaining

$$
\hat{\phi} \approx-\epsilon^{3 / 2} \eta \phi
$$

(with $\eta=T_{e} / T_{i}$ ). This demonstrates that the adiabatic response of the trapped ions at the island separatrix has a feedback on the electrostatic potential (the minus sign shows that this correction lowers the value of the potential therein). Numerical simulations show that this correction has the effect of smoothing the potential profile, which in turn leads to a smoothing of the discontinuity in the radial electric field at the island separatrix. In Fig. 3 a comparison between the radial derivative of $\phi_{0}$ (see Eq.(8)) with a GKW numerical simulation is presented. The abrupt "spike" at the island separatrix introduced by a Heaviside step function which characterises the analytical model is not reproduced in our numerical simulations (we checked that this is a convergent result). Fig.4 shows the variation of the peak electric field at the island separatrix for different values of $\eta$. It is evident that for hotter electrons, a higher $\hat{\phi}$ is needed in 
order to ensure quasineutrality, and therefore, consistently with Eq.(12), the smoothing of the potential profile at the island separatrix is much more efficient for higher $\eta$. Furthermore, it is interesting to note, as shown in Fig.5, that such smoothing also becomes less effective for smaller island widths.

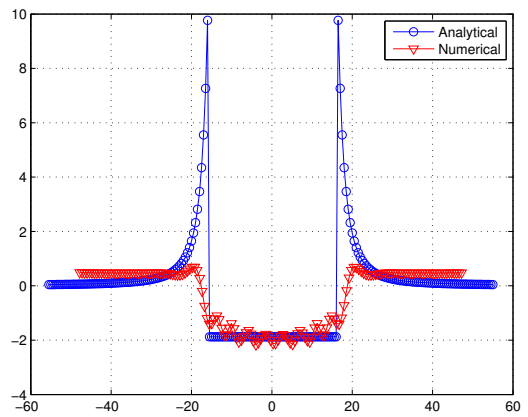

Figure 3: Comparison for of the radial profile of the radial electric field at the island O-point between the analytical formula Eq.(8) and the numerical simulations for $\omega=0.04$ in arbitrary units.

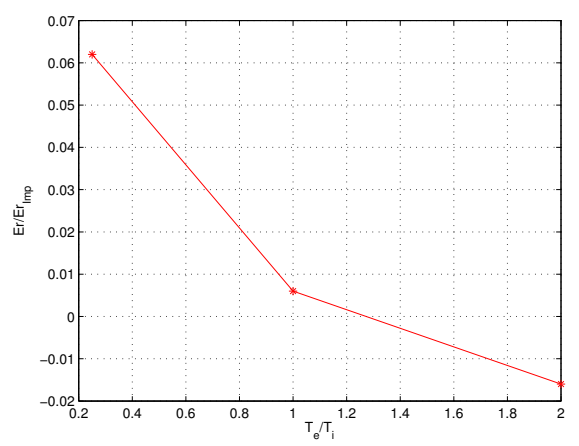

Figure 4: Value of the "peak" of the radial electric field at the island separatrix (see Fig.3) normalized to the corresponding electric field associated to $\phi_{0}$ at $\omega=-0.02$ and $\hat{w}=16$ for various $T_{e} / T_{i}$. The negative sign indicates a smooth increase of the $E_{r}$ radial profile towards the value inside the island (cf. Fig.3), without any peak and any abrupt change of sign. 


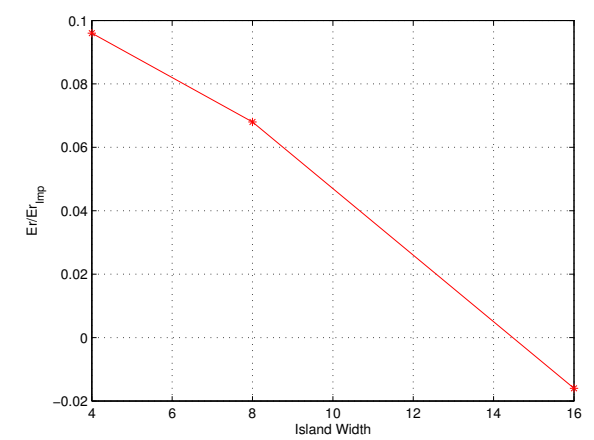

Figure 5: Value of the "peak" of the radial electric field at the island separatrix (see Fig.3)normalized to the corresponding electric field associated to $\phi_{0}$ at $\omega=-0.02$ and $T_{e} / T_{i}=2$ for various island widths (normalized to the thermal ion Larmor radius). The smoothing at the separatrix is less effective when the island is smaller. With the parameters employed in our simulations, the ion banana width amounts to $\rho_{b}=3.44 \rho_{L}$.

\section{Effects on the Polarization Current}

The modification of the electrostatic potential discussed in the previous section has a significant impact on the island polarization current. As mentioned in the introduction, the polarization current is often included in the modelling of NTMs as a possible explanation of the threshold for the excitation of a island found in many experiments [18]. In order to concentrate on the polarization current alone, in this section we still neglect the equilibrium gradients.

Strictly speaking, the polarization current involves a neoclassical and a classical contribution. The first one, which is expected to be the larger one [11], is linked to the fact that trapped particles move with respect to the magnetic island by virtue of their toroidal precession caused by the island electric field (with frequency $\omega_{E, t r}=c E_{r} / B_{p}$ ) [31]. Therefore, they experience a timevarying electric field (in a Lagrangian sense), which lets them drift across the perturbed magnetic surface, this effect being larger for ions than for electrons and thus generating a perpendicular current. The second mechanism (classical) is analogous to the first one, but it is linked to the different response of ions and electrons when the scale of variation of the potential is compatible with the ion Larmor radius [21]. The discontinuous derivative of $E_{r, 0}=-\mathrm{d} \phi_{0} / \mathrm{d} x$ at the island separatrix leads to singular gradients in the perpendicular polarization current (both classical and neoclassical), which in view of the quasineutrality constraint $\nabla_{\|} J_{\|}=-\nabla_{\perp} \cdot \mathbf{J}_{\perp}$ correspond to a parallel current $J_{\|}$[12]. We recall that the perturbed parallel current is what ultimately affects the island 
stability, as in steady state [2]

$$
\sum_{ \pm} \int_{-1}^{\infty} \mathrm{d} \Omega \oint \mathrm{d} \xi \frac{J_{\|}(w, \omega) \cos \xi}{\sqrt{\Omega+\cos \xi}}=\frac{c \Delta^{\prime}}{4} \tilde{\psi}
$$

where $\tilde{\psi}=-R \hat{A}_{\|}$and the sum over \pm corresponds to the two different regions, $x>0$ and $x<0$, where the integration should be carried out.

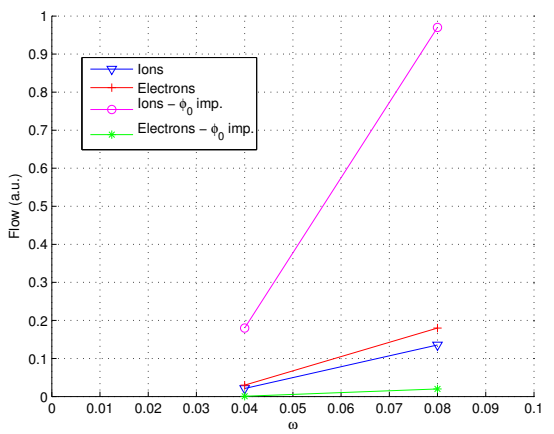

Figure 6: Peak of the perpendicular ion and electron flow associated to the classical polarization current (defined as $\int \mathrm{d}^{3} v \tilde{f}_{i, e}(X)\left\langle\mathbf{v}_{E}\right\rangle \cdot \nabla \Omega[20]$, with angular brackets denoting gyroaverage) at $\xi=-\pi / 2$ (i.e. between O- and X-point, cf. Fig.7) with imposed electrostatic potential $\phi_{0}$ (circles and stars) and with self-consistent electrostatic potential (triangles and crosses) for various frequencies and $\hat{w}=3$. Note the large difference between the self-consistent cases and the imposed $\phi_{0}$ cases even for this small island size. Note also the quadratic dependence on $\omega$ for the ions. All runs have been performed with the same spatial resolution.

In absence of equilibrium gradients, the polarization current (both classical and neoclassical) is characterized by a quadratic dependence on the island rotation frequency $\omega$ (it is proportional to the time derivative $\mathrm{d} / \mathrm{d} t \propto \omega$ of the $\mathbf{E} \times \mathbf{B}$-drift, which is itself proportional to $\omega)$. Furthermore, the perpendicular polarization drift, as mentioned, is much larger for ions than for electrons (the subsequent closure parallel current, which is sometimes referred to as "polarization current" as well, is on the contrary mostly carried by electrons). In Fig.6, the classical polarization current calculated in GKW simulations by replacing the self-consistent potential with $\phi_{0}$ (Eq.(8) and (9)) is compared to the one resulting from self-consistent runs. The difference between the two current peaks at the island separatrix corresponds to a factor of around 10 . According to our interpretation, this is a consequence of the smoothing of the potential profile discussed in section 3 . In addition, only when the potential $\phi_{0}$ is imposed, the signal can be clearly observed to be larger for ions than for electrons. The fact that the polarization current spike at the island separatrix is overestimated when Eq.(8) is employed has been already suggested in 
Ref.[32], where the effect of diffusion across the island separatrix in slab geometry has been analyzed. However, the smoothing due to the adiabatic response of trapped particles seems to be even more effective than the diffusion to reduce the influence of the polarization current.

Similarly, the neoclassical polarization current undergoes the same depletion when the self-consistent calculation of the potential is employed. In our numerical simulations (see Fig.7) there is no dominant signal in the neoclassical perpendicular flows (defined as $\int \mathrm{d}^{3} v \delta f_{j} \mathbf{v}_{D, j}$, where $\mathbf{v}_{D}$ indicates the magnetic drift velocity and $j$ labels the species) which exhibits a quadratic dependence on the island rotation frequency which is at the same time much larger for ions than for electrons. Again, we interpret this strong depletion to be connected with the smoothing of the electrostatic potential at the island separatrix. A quadratic scaling of the neoclassical ion flux starts to be observed for island rotation frequencies of the order of the ion bounce frequency (i.e. $\omega \approx 0.44$ in our normalized units), which does not represent a realistic value from the physical point of view.
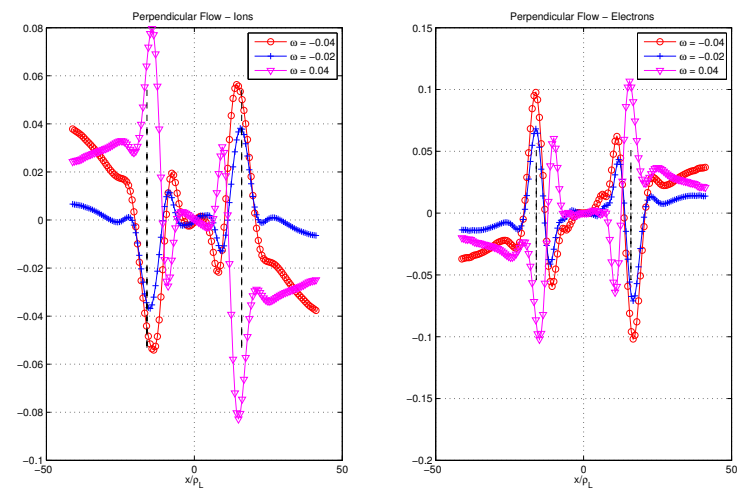

Figure 7: Perpendicular profile of the $s$-averaged ion and electron neoclassical flow $\int \mathrm{d}^{3} v \delta f_{j} \mathbf{v}_{D, j}$ (where $j$ labels the species and which is expected to be dominated by the neoclassical polarization current when $\phi_{0}$ is employed, see [11]) at $\xi=-\pi / 2$ (i.e. between the O-Point and the X-point) for various values of the island rotation frequency.

The dominant signal visible in the neoclassical perpendicular current in Fig.7, exhibiting a linear dependance on $\omega$, is most probably associated to the adiabatic perturbation of the density at the island separatrix.

Summarizing, for realistic values of $\omega$, no signal identifiable as the neoclassical polarization current is found in the simulations, although the classical contribution is stil visible (at a significantly reduced value than predicted from Eq.(8)). 


\section{Electrostatic Potential - Inclusion of Equilib- rium Pressure Gradients}

In this section, we discuss the effects of the adiabatic response of trapped particles both on the electrostatic potential and on the flattening of the density profile when equilibrium gradients are included. We recall that a reduced degree of flattening inside the island region corresponds to a reduced bootstrap drive in the island dynamics.

When no magnetic island is present, the equilibrium diamagnetic drift $\left(v_{*, j}=c \nabla p_{j} / n_{j} q_{j} B\right.$ for the $j$-th species, where $p_{j}$ indicates the pressure and $q_{j}$ the charge) is in the $\nabla \zeta$-direction (which around the rational surface can be approximated with $\nabla \xi$ ) with opposite sign for electrons and ions. Let $\omega_{*, e, i}$ be the corresponding equilibrium frequencies for electrons $(e)$ and ions $(i)$, respectively. In presence of a magnetic island, the total rotation frequency of the electrons $\omega_{T O T, e}$

$$
\omega_{T O T, e}=\omega_{E}+\omega_{*, e}+\tilde{\omega}_{*, e},
$$

(with $\tilde{\omega}_{*}$ being associated to the perturbation) is expected to match the island rotation frequency, as electrons tend to remain "frozen", with a high degree of approximation, to the perturbed magnetic surfaces. In the limit of complete flattening, the total diamagnetic rotation of the species $\omega_{*, e, i}+\tilde{\omega}_{*, e, i}$ drops to zero. The electrostatic potential therefore has the role of providing an $\mathbf{E} \times \mathbf{B}$ rotation in order to let the plasma inside the island rotate together with it, as discussed before, i.e. it leads to $\omega_{T O T, e, i}=\omega_{E}=\omega$. Turning to our numerical GKW simulations, we indeed observe that for islands rotating in the ion diamagnetic direction (i.e. $\omega<0$ ) the density perturbation following the adiabatic law Eq.(10) leads to a high degree of density flattening even for small island widths, cf. Ref.[17] and the discussion at the end of this section. This of course implies $\omega_{*, e, i}+\tilde{\omega}_{*, e, i} \approx 0$, and thus, in order to let the plasma rotate together with the island, the potential therein should look similar to $\phi_{0}$ (at least far away from the separatrix), i.e. $\omega_{E} \approx \omega$. Indeed, this is what occurs, and the results are visible in Fig.8, which shows $\omega_{T O T, e, i}$ as a function of the radial coordinate for various values of the island width. Note that outside the island, the value of $\omega_{T O T, e, i}$ corresponds to the respective equilibrium diamagnetic frequencies, as the effect of the perturbations connected to the island rapidly vanishes in the external region. Note also that the "peaks" visible around the island separatrix are associated to the very steep pressure gradients immediatly outside the island.

When the island rotates in the electron diamagnetic direction (i.e. $\omega>0$ ), the phenomenology is more complicated, as shown in Fig.9. In fact, the density perturbation associated with the adiabatic response now opposes the flattening [17]. This means that the sum $\omega_{*, e, i}+\tilde{\omega}_{*, e, i}$ is no longer zero. Thus, the potential that develops to force the electrons to rotate with the island inside it can differ dramatically from Eq.(8), where density flattening was assumed. In 

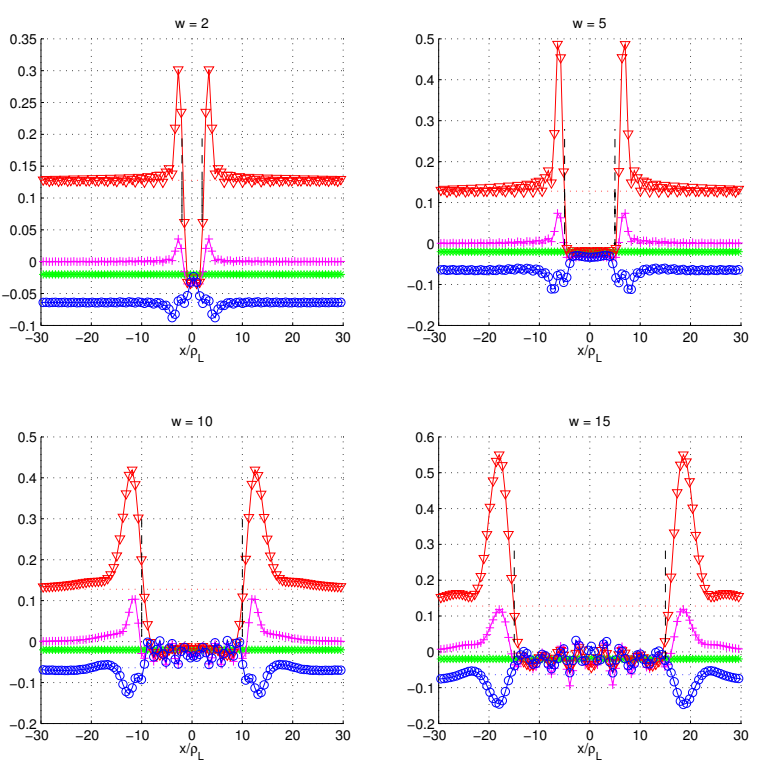

Figure 8: Radial profile of the total rotation frequency $\omega_{T O T}$ for electrons (red triangles) and ions (blue circles) for islands rotating in the ion diamagnetic direction ( $\omega=-0.02)$ showing that the sum of the electric and diamagnetic contributions inside the island matches the island rotation frequency (green stars) for different island widths $w$. The rotation frequency $\omega_{E}$ (magenta crosses) is represented as well. In the considered simulations, the value of the logarhitmic gradients $(R / L n=$ $\left.R / L T_{i}=R / L T_{e}=1.5\right)$ has been kept low in order to exclude turbulent effects, while $T_{e} / T_{i}=2$. Note that, in the simulations, the total width of the computational domain amounts to $\approx 160 \rho_{L}$, but here, for clearness, only the island region has been represented.

particular, in the case of no flattening and $\omega<\omega_{*, e}$, the electric field calculated in self-consistent simulations points in the opposite direction to that derived from Eq.(8). In Bergmann et al. [17] the impact of the adiabatic response on the depletion of the equilibrium bootstrap current especially in small islands has been analyzed. In that paper, the numerical investigation was performed with an imposed electrostatic potential $\phi_{0}$ rather than with a self-consistent one, and in fact the authors observed that the quasineutrality constraint was not satisfied under those condition. Thus, they suggested a modification of $\phi_{0}$ based on simple physical assumptions, namely

$$
\phi_{\text {Bergmann }}=\frac{T_{i}}{T_{i}+T e}\left(1-\frac{\omega_{*, e}}{\omega}\right) \phi_{0} .
$$

Eq.(15) agrees with the behaviour of the self-consistent electrostatic potential 

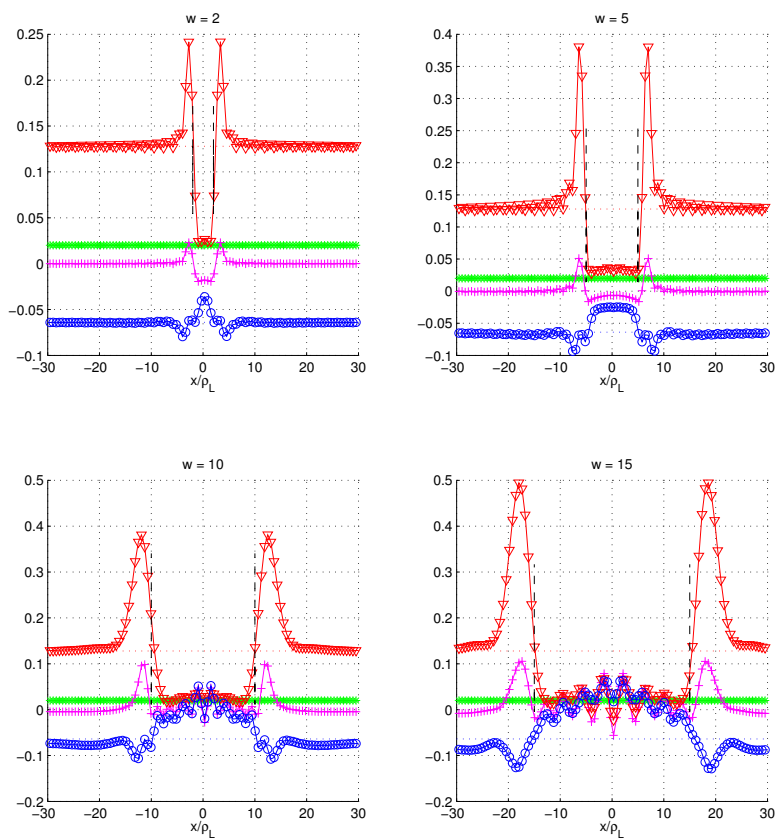

Figure 9: The same as in Fig. 8 for islands rotating in the electron diamagnetic direction $(\omega=0.02)$. In contrary to Fig. 8 , the adiabatic response opposes the flattening, and therefore the electrostatic potential develops in order to let electrons rotate with the island. For small widths, ions are not able to follow the island.

found in our numerical simulations. This confirms the validity of the conclusions of Ref.[17], according to which small islands rotating in the electron diamagnetic direction exhibit an enhanced stability against the neoclassical drive. We note, for completeness, that the same expression as Eq.(15) for the electrostatic potential has been derived by Smolyakov [30] in slab geometry in the limit $w \ll \rho_{L}$. Moreover, in Ref.[17] a limited flattening of the electron density profile for islands rotating in the electron diamagnetic direction was observed. This result is completely consistent with our simulations, as can be observed in Fig.10, where the total density gradient as a function of the island frequency is represented.

\section{Conclusions}

We have carried out a gyrokinetic numerical investigation of the electrostatic potential associated with an imposed rotating magnetic island. The numerical 


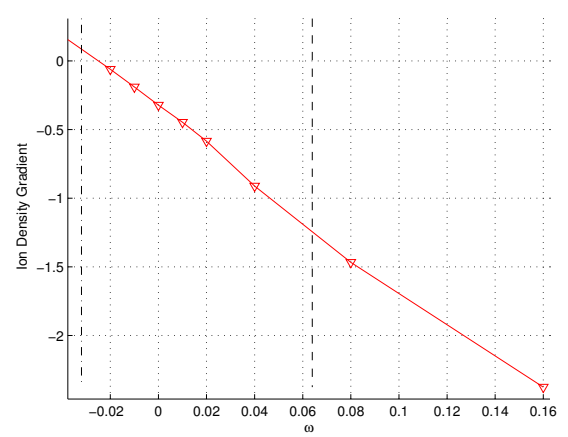

Figure 10: Normalized ion density gradient at the island O-point for an island with $\hat{w}=3$ for various values of the island rotation frequency. The vertical dasheddotted line identifies the ion diamagnetic frequency, while the dashed one the electron diamagnetic frequency. The normalized equilibrium density gradient has been set to $\rho_{L} n_{0}^{-1} \mathrm{~d} n_{0} / \mathrm{d} x=-1.5$.

tool employed allows us to retain a large number of physical effects, including FLR effects and the trapped particle contribution. The self-consistent gyrokinetic Poisson equation, in particular, exempts us from adopting any assumption on the electrostatic potential. Simulations performed with flat equilibrium gradients allowed us to identify an adiabatic response of the trapped ions around the island separatrix, where the scales of variation of the electrostatic potential are short with respect to the orbit width. Such perturbed density has in turn an effect on the electrostatic potential, as the discontinuity at the island separatrix which characterises the models based on Eq.(8) is found to be smoothed. Here, the smoothing is found self-consistently and does not need any ad-hoc modelling $[20,32]$. The differences between the self-consistent electrostatic potential and the analytical $\phi_{0}$ reveal their importance when the corresponding polarization currents, classical and neoclassical, are considered. It is evident that the magnitude of the classical polarization current is overestimated when $\phi_{0}$ is employed, while the neoclassical polarization current is not even visible when the self-consistent electrostatic potential is considered, at least when realistic values of $\omega$ are used. Thus, it is very likely that other contributions play a more significant role than polarization current in determining the stability of small islands.

When the equilibrium gradients are considered, the adiabatic response is shown to reduce the flattening in the island region if the island rotates in the electron diamagnetic direction. In those cases, the electron equilibrium diamagnetic rotation, in particular for small islands, is preserved to a large degree, and the subsequent electrostatic potential, which should ensure $\omega=$ $\omega_{E}+\omega_{*, e}+\tilde{\omega}_{*, e}$, deviates significantly from the standard analytic potential 
$\phi_{0}$ of Eq.(8). Our simulations also confirm the validity of previous results [17] about the modifications to the neoclassical drive in small islands by virtue of the ion adiabatic response.

It is important however to stress that in our simulations the island rotation frequency $\omega$ remains an imposed quantity. An investigation of the "natural" island rotation frequency can be performed via a parametric study of the torque acting on the island region [33]. This study will be considered in a separate publication.

\section{Acknowledgments}

The authors gratefully acknowledge the precious technical support of Dr. Alberto Bottino and Dr. Andrew P. Snodin. The numerical simulations presented in this paper have mostly been performed on the HPC-FF machines in Jülich (Germany).

\section{References}

[1] H. P. Furth, J. Killeen, M. N. Rosenbluth Phys. Fluids 6459 (1963)

[2] P. H. Rutherford, Phys. Fluids 161903 (1973)

[3] R. Carrera, R. D. Hazeltine, M. Kotschenreuther, Phys. Fluids 29899 (1986)

[4] W. X. Qu and J. D. Callen University of Wisconsin Plasma Report No UWPR 85-5 (1985)

[5] Z. Chang et al., Phys. Rev. Letters 744663 (1995)

[6] O. Sauter et al., Phys. Plasmas 41654 (1997)

[7] R. Fitzpatrick, Phys. Plasmas 2825 (1995)

[8] E. Poli et al., Phys. Rev. Letters 88075001 (2002)

[9] E. Poli et al., Plasma Phys. Control. Fusion 4571 (2003)

[10] A. I. Smolyakov et al., Phys. Plasmas 21581 (1995)

[11] H. R. Wilson et al., Phys. Plasmas 3248 (1996)

[12] F. L. Waelbroeck, R. Fitzpatrick, Phys. Rev. Letters 781703 (1997)

[13] A. B. Mikhailovskii, Contrib. Plasma Phys. 43125 (2003)

[14] S. V. Konovalov et al., Doklady Physics 47488 (2002) 
[15] E. Poli, A. Bergmann and A. G. Peeters, Phys. Plasmas 94205001 (2005)

[16] M. Siccinio, E. Poli, Plasma Phys. Control. Fusion 51075005 (2009)

[17] A. Bergmann, E. Poli and A. G. Peeters, Phys. Plasmas 16092507 (2009)

[18] O. Sauter et al, Plasma Phys. Control. Fusion 52025002 (2010)

[19] F. L. Waelbroeck J. W. Connor, H. R.Wilson, Phys. Rev. Letters 87 215003-1 (2001)

[20] M. James, H. R. Wilson, Plasma Phys. Control. Fus. 481647 (2006)

[21] J. W. Connor, H. R. Wilson, Phys. Plasmas 24275 (1995)

[22] A. G. Peeters et al., Comp. Phys. Comm. 1802649 (2009)

[23] S. Cowley, R. Kulsrud and R. Sudan, Phys. Fluids B 32767 (1991)

[24] M. Beer, S. Cowley and G. Hammet, Phys. Plasmas 22687 (1995)

[25] A. Brizard, Phys. Plasmas 41541 (1988)

[26] H. Sugama, Phys. Plasmas 7, 466 (2000)

[27] S. Hamada, Nucl. Fusion 223 (1962)

[28] W. A. Hornsby et al., Phys. Plasmas 17092301 (2010)

[29] E. Poli et al., Plasma Phys. Control. Fus. 52124021 (2010)

[30] A. I. Smolyakov, Plasma Phys. Control. Fusion 35657 (1993)

[31] F. L. Hinton and J. A. Robertson, Phys. Fluids 271243 (1984)

[32] M. James, H. R. Wilson, Plasma Phys. Control. Fus. 52075008 (2010)

[33] F. Militello et al., Phys. Plasmas 15050701 (2008) 